\title{
Model-independent analysis of U-spin breaking in non-leptonic B-decays
}

\author{
Martin Jung* \\ Theoretische Physik 1, Fachbereich Physik, Universität Siegen \\ D-57068 Siegen, Germany \\ Instituto de Física Corpuscular, CSIC-Universitat de València, \\ Apartado de Correos 22085, E-46071 Valencia, Spain. \\ E-mail: jung@hep.physik.uni-siegen.de \\ Thomas Mannel \\ Theoretische Physik 1, Fachbereich Physik, Universität Siegen \\ D-57068 Siegen, Germany \\ E-mail: mannel@hep.physik.uni-siegen.de
}

\begin{abstract}
Flavour symmetries represent a powerful tool in analysing model-independently hadronic matrix elements. Going beyond isospin symmetry results, however, in a large uncertainty related to the symmetry breaking due to the relatively large strange quark mass. Considering the $U$-spin subgroup of flavour SU(3), its breaking is analysed on a group theoretical basis. Due to the simple behaviour of the weak effective hamiltonian under $U$-spin and the unique structure of the breaking terms, such a group theoretical analysis leads to a manageable number of parameters. In view of the enormous amount of data expected from $\mathrm{LHCb}$ and a possible Super B factory, this data driven method provides a way of gathering information on hadronic matrix elements in general and $U$-spin breaking in particular. As a first application, the pair of decays $B^{-} \rightarrow J / \psi K^{-}$ and $B^{-} \rightarrow J / \psi \pi^{-}$is discussed.
\end{abstract}

International Workshop on Effective Field Theories: from the pion to the upsilon 2-6 February 2009

Valencia, Spain

\footnotetext{
*Speaker.
} 


\section{Introduction}

Non-leptonic decays of bottom hadrons play an important role in the investigation of CP violation. While this effect has been established in non-leptonic Kaon decays, and the CKM mechanism of $\mathrm{CP}$ violation is consistent with what is seen in non-leptonic $B$ meson decays, the observations cannot be easily linked to the fundamental parameters. The reason for this is a lack of a reliable method to compute the amplitudes of non-leptonic decays, which are given by matrix elements of an effective interaction expressed in terms of a combination of four-quark operators between meson states. The QCD dynamics turn out to be so complicated, that currently neither semi-perturbative methods like QCD Factorization, SCET or pQCD, nor lattice calculations yield reliable and precise predictions.

While non-leptonic decays are a nice laboratory for studying QCD methods, the road to precise predictions for $\mathrm{CP}$ violation in non-leptonic $B$ decays seems to be the use of flavour symmetries, supplemented by the enormous amount of data expected from LHC and the (Super) flavour factories. From the current perspective this will remain true for some time, until a qualitative breakthrough is achieved in the field of QCD methods. The main problem with flavour symmetries is that they hold only approximately. The usual starting point is flavour $S U(3)$, which suffers from a substantial breaking due to the sizable mass of the strange quark. However, its isospin subgroup is a very good symmetry, which safely can be assumed to be unbroken. Hence one may consider the other two possibilities to identify $S U(2)$ subgroups of flavour $S U(3)$, which run under the names $U$-spin and $V$-spin. Among these, the generators of $U$-spin (under which the $d$ and the $s$ quark form a fundamental doublet) commute with the charge operator, which makes this subgroup particularly interesting with respect to electroweak interactions. On the other hand, $U$-spin symmetry is broken at the same level as the full flavour $S U(3)$ due to the splitting $m_{s}-m_{d}$. However, this breaking has a simple structure and can be readily included by a spurion analysis. In this talk the corresponding formalism is presented, along with an example application to the pair of decays $B^{-} \rightarrow J / \psi K^{-}$and $B^{-} \rightarrow J / \psi \pi^{-}$.

\section{U-spin and its breaking}

$U$-spin is an $S U(2)$ subgroup of the full flavour $S U(3)$ symmetry group, in which the $d$ and the $s$ quark form a doublet. A priori, $U$-spin is as badly broken as the full $S U(3)$, since the masses of the two quarks are substantially different:

$$
\Delta m \equiv m_{s}-m_{d} \sim \Lambda_{\mathrm{QCD}},
$$

where $\Lambda_{\mathrm{QCD}}$ is the nonperturbative QCD scale.

This results in a breaking, since the relevant mass term in the Lagrangian reads

$$
\begin{aligned}
\mathscr{L}_{\text {smass }} & =m_{d} \bar{d} d+m_{s} \bar{s} s=\frac{1}{2}\left(m_{s}+m_{d}\right)(\bar{d} d+\bar{s} s)+\frac{1}{2} \Delta m(\bar{s} s-\bar{d} d) \\
& =\frac{1}{2}\left(m_{s}+m_{d}\right) \bar{q} q+\frac{1}{2} \Delta m \bar{q} \tau_{3} q,
\end{aligned}
$$

where

$$
q=\left(\begin{array}{l}
d \\
s
\end{array}\right)
$$


denotes the $U$-spin quark doublet. Thus the breaking term can be described as a triplet spurion

$$
\mathscr{H}_{\text {break }}=\frac{1}{2} \Delta m \tau_{3}=\varepsilon B_{0}^{(1)},
$$

where $\varepsilon \sim \Delta m$ and $B_{0}^{(1)}$ is an irreducible tensor-operator with $j=1$ and $j_{3}=0$.

If we consider a matrix element of some operator $\mathscr{O}(x)$, which can be decomposed into irreducible tensor-operators of $U$-spin , we may calculate $U$-spin breaking to leading order by evaluating

$$
\langle\tilde{f}|\mathscr{O}(0)| \tilde{i}\rangle=\langle f|\mathscr{O}(0)| i\rangle+(-i) \int d^{4} x\left\langle f\left|T\left[\mathscr{O}(0) H_{\text {break }}(x)\right]\right| i\right\rangle+\ldots,
$$

where the states $\tilde{f}$ and $\tilde{i}$ include the breaking term, while the states $f$ and $i$ are $U$-spin symmetric. A general analysis of $U$-spin breaking can be perfomed by a group theoretical analysis of the breaking term, by decomposing the $T$ product of the operator $\mathscr{O}$ with $H_{\text {break }}$ into irreducible tensor operators $T_{j_{3}}^{(j)}$ of $U$-spin .

The simplest, non-trivial case emerges if the operator $\mathscr{O}$ is an $U$-spin doublet, which we denote by $\mathscr{O}_{j_{3}}^{(1 / 2)}$. In this case, the last term in (2.3) decomposes into

$$
\begin{aligned}
(-i) \int d^{4} x T\left[\mathscr{O}_{ \pm 1 / 2}^{(1 / 2)}(0) H_{\text {break }}(x)\right] & =(-i \varepsilon) \int d^{4} x T\left[\mathscr{O}_{ \pm 1 / 2}^{1 / 2}(0) B_{0}^{(1)}(x)\right] \\
& =\sqrt{\frac{2}{3}}\left[K_{ \pm 1 / 2}^{(3 / 2)} \mp \sqrt{\frac{1}{3}} K_{ \pm 1 / 2}^{(1 / 2)}\right] .
\end{aligned}
$$

Aside from the trivial example of the currents $j=\bar{u} \Gamma q, q=d, s$ also the effective weak hamiltonian for $B$ decays is a pure $U$-spin doublet, even if electroweak penguins are included. The latter is true due to the fact that the $s$ and the $d$ quark carry the same electroweak quantum numbers. Thus from the group theoretical point of view we may decompose the weak effective Hamiltonian into its irreducible tensor components according to

$$
\begin{aligned}
H_{\mathrm{eff}}^{\Delta C= \pm 1} & =\frac{4 G_{F}}{\sqrt{2}}\left[V_{c b} V_{u d}^{*} P_{1 / 2}^{(1 / 2)}+V_{c b} V_{u s}^{*} P_{-1 / 2}^{(1 / 2)}\right], \\
H_{\mathrm{eff}}^{\Delta C=0} & =\frac{4 G_{F}}{\sqrt{2}}\left[V_{c b} V_{c d}^{*} Q_{1 / 2}^{(1 / 2)}+V_{u b} V_{u d}^{*} R_{1 / 2}^{(1 / 2)}+V_{c b} V_{c s}^{*} Q_{-1 / 2}^{(1 / 2)}+V_{u b} V_{u s}^{*} R_{-1 / 2}^{(1 / 2)}\right]
\end{aligned}
$$

where the operators $P_{j_{3}}^{(1 / 2)}, Q_{j_{3}}^{(1 / 2)}$ and $R_{j_{3}}^{(1 / 2)}$ are renormalization group invariant combinations of four-quark operators.

In the following we shall use this group theoretical decomposition to discuss $U$-spin and its breaking in $B$ decays and the corresponding $\mathrm{CP}$ asymmetries. To this end, we have to identify the $U$-spin multiplets of hadronic states. Starting from the definiton of the fundamental quark doublets (we use the same sign convention as in [1]),

$$
\left[\begin{array}{l}
|d\rangle \\
|s\rangle
\end{array}\right]=\left[\begin{array}{l}
\left|\frac{1}{2}+\frac{1}{2}\right\rangle \\
\left|\frac{1}{2}-\frac{1}{2}\right\rangle
\end{array}\right], \quad\left[\begin{array}{l}
|\bar{s}\rangle \\
|\bar{d}\rangle
\end{array}\right]=\left[\begin{array}{r}
\left|\frac{1}{2}+\frac{1}{2}\right\rangle \\
-\left|\frac{1}{2}-\frac{1}{2}\right\rangle
\end{array}\right],
$$

we obtain for the decaying $B$ mesons

$$
\left|B^{+}\right\rangle=|u \bar{b}\rangle=|0,0\rangle, \quad\left[\begin{array}{l}
\left|B^{0}\right\rangle=|(d \bar{b})\rangle \\
\left|B_{s}\right\rangle=|(s \bar{b})\rangle
\end{array}\right]=\left[\begin{array}{l}
\left|\frac{1}{2},+\frac{1}{2}\right\rangle \\
\left|\frac{1}{2},-\frac{1}{2}\right\rangle
\end{array}\right] .
$$


The mesons in the final state can also be decomposed along these lines. Charged (Neutral) B mesons being $U$-spin singlets (doublets), the possible final states have $U=1 / 2,3 / 2(U=0,1,2)$, where states with $U=3 / 2(2)$ can only be reached by a $U$-spin breaking transition, and are thus absent in [1]. In some cases, the corresponding parameters are in principle extractable from data [2]. In most cases, however, this analysis results in more parameters than available observables. Therefore one has again to rely on theoretical arguments to reduce the number of parameters. One option to be employed later is to use power counting arguments for certain decay topologies, and neglect the $U$-spin breaking corrections for amplitudes expected to be small.

Regarding the $U$-spin limit, there is one relation for decays $B \rightarrow f_{1}, B \rightarrow f_{2}$, related by exchanging all $d, s$ quarks in the process [3], following from the Wigner-Eckart theorem and the relation $\operatorname{Im}\left(V_{c b} V_{c d}^{*} V_{u b}^{*} V_{u d}\right)=-\operatorname{Im}\left(V_{c b} V_{c s}^{*} V_{u b}^{*} V_{u s}\right)$ :

$$
\Delta \Gamma_{1} \equiv \Gamma\left(B \rightarrow f_{1}\right)-\Gamma\left(\bar{B} \rightarrow \bar{f}_{1}\right)=-\Delta \Gamma_{2},
$$

or, in terms of observables,

$$
A_{\mathrm{CP}}\left(B \rightarrow f_{1}\right) \bar{\Gamma}\left(B \rightarrow f_{1}\right)=-A_{\mathrm{CP}}\left(B \rightarrow f_{2}\right) \bar{\Gamma}\left(B \rightarrow f_{2}\right) .
$$

This relation espespially implies opposite signs for the $\mathrm{CP}$ asymmetries, and may serve as a first test for the amount of $U$-spin breaking in the decay pair considered.

\section{Application to $\mathbf{B} \rightarrow \mathbf{J} / \psi \mathbf{K}, \pi$}

As a first application, the decays $B \rightarrow J / \psi(K$ or $\pi)$ are considered, which are under the simplest cases of $\Delta C=0$ from the group-theoretical point of view, because of $B^{-}$and $J / \psi$ being $U$-spin singlets. This results in

$$
\left\langle B^{-}\left|\mathscr{H}_{e f f}\right| J / \psi K^{-}\left(\pi^{-}\right)\right\rangle=\sum_{q=u, c} \lambda_{q s / d}\left(A_{q, 1 / 2} \pm A_{q, 1 / 2}^{\varepsilon}\right) .
$$

The analysis is based on the data shown in table 1.

\begin{tabular}{|l|l|l|}
\hline Decay & $B R / 10^{-4}$ & $A_{C P}$ \\
\hline$B^{-} \rightarrow J / \psi K^{-}$ & $10.07 \pm 0.035$ & $0.017 \pm 0.016(*)$ \\
$B^{-} \rightarrow J / \psi \pi^{-}$ & $0.49 \pm 0.06(*)$ & $0.09 \pm 0.08$ \\
\hline
\end{tabular}

Table 1: Measurements for the decays $B^{-} \rightarrow J / \psi K / \pi$, data taken from the PDG [4]. (*): Error enhanced by PDG because of inconsistent measurements.

As a first step, it is checked for $U$-spin violation by testing the $U$-spin relation (2.9). Inserting the data from table 1 and neglecting tiny phase space differences results in

$$
\left(A_{C P} \times B R\right)_{B^{-} \rightarrow J / \psi K^{-}}+\left(A_{C P} \times B R\right)_{B^{-} \rightarrow J / \psi \pi^{-}}=0.22 \pm 0.17,
$$

adding errors simply in quadrature. This result is not significant and a real test may only be performed, if at least one of the asymmetries is measured significantly different from zero. 
In many applications naive factorization has been applied, which allows to include at least the factorizable part of $U$-spin breaking. In this picture one expects the ratio of branching ratios to be given only in terms of CKM factors and the ratio of form factors. One gets the theoretical prediction

$$
\frac{B R\left(B^{-} \rightarrow J / \psi K^{-}\right)}{B R\left(B^{-} \rightarrow J / \psi \pi^{-}\right)} \sim\left(\frac{F^{B \rightarrow K}\left(M_{J / \psi}^{2}\right)}{F^{B \rightarrow \pi}\left(M_{J / \psi}^{2}\right)}\right)^{2}\left|\frac{V_{c b}^{*} V_{c s}}{V_{c b}^{*} V_{c d}}\right|^{2}=33.9 \pm 6.1,
$$

where the form factor ratio is taken from QCD sum rule calculations [5] and scaled to $q^{2}=m_{J / \psi}^{2}$ with aid of a simple BK ansatz[6]. This has to be contrasted with the experimental number

$$
\frac{B R\left(B^{-} \rightarrow J / \psi K^{-}\right)}{B R\left(B^{-} \rightarrow J / \psi \pi^{-}\right)}= \begin{cases}19.2 \pm 1.5 & \text { (measurement of the ratio) } \\ 21.4 \pm 1.9 & \text { (combined single measurements) }\end{cases}
$$

The sizable discrepancy indicates the well known fact that these decays have large non-factorizable contributions.

On the other hand, the data in table 1 are not sufficient to allow a fit to the general grouptheoretical expressions. Hence additional assumptions are necessary. The amplitude proportional to $\lambda_{u d / s}=V_{u b} V_{u d / s}^{*}$ is expected to be small compared to the one proportional to $\lambda_{c d / s}=V_{c b} V_{c d / s}^{*}$ because its tree contribution has only penguin matrix elements. Hence the breaking corrections to this amplitude will not be taken into account This results in the parametrization

$$
\begin{aligned}
& <B^{-}\left|\mathscr{H}_{e f f}\right| J / \psi K^{-}>=N_{J / \psi K}\left(1+x_{\varepsilon}+\lambda^{2} /\left(1-\lambda^{2}\right) e^{-i \gamma} r_{0} e^{i \phi_{0}}\right), \\
& <B^{-}\left|\mathscr{H}_{e f f}\right| J / \psi \pi^{-}>=\frac{\lambda}{1-\lambda^{2} / 2} N_{J / \psi K}\left(-1+x_{\varepsilon}+e^{-i \gamma} r_{0} e^{i \phi_{0}}\right),
\end{aligned}
$$

where the normalization factor $N_{J / \psi K}$ is chosen such that $N_{J / \psi K}^{2}=B R\left(B^{-} \rightarrow J / \psi K^{-}\right)$in absence of $U$-spin breaking and penguin effects. Furthmermore, the ratio $r_{0}$ represents all contributions proportional to $\lambda_{u d, s}$ in the $U$-spin limit, and includes a factor of $R_{u}=\left|V_{u b} V_{u d}^{*} / V_{c b} V_{c d}^{*}\right|$ from the CKM elements. It is therefore expected to be of order $R_{u} \times \lambda$, with $\lambda$ representing the penguin suppression factor. Finally, $x_{\varepsilon}$ parametrizes the $U$-spin breaking in the leading contribution, again normalized to $N_{J / \psi K}$.

At this stage, the number of observables does still not suffice to allow for a determination of all fit parameters. However, as only one observable is missing, one can examine the resulting correlations. In addition, one may use the fact that isospin can be assumed to hold exactly at this level of approximation and use the neutral decays in addition, see [2].

The inputs from the CKM fit are [7]

$$
\lambda=0.2252 \pm 0.0008 \text { and } \gamma=\left(66.8_{-3.8}^{+5.4}\right)^{\circ}
$$

where the lower uncertainty of $\gamma$ has been slightly enhanced to reflect the non-gaussian behaviour of the distribution in a conservative way.

The fit results for the $U$-spin breaking parameters are shown in fig. 1. The fit shows two distinct solutions, one of which has $\phi_{0} \sim 0$ while the other one has $\phi_{0} \sim \pi$. As the solutions interfere in the fit and make it unstable, we perform two separate fits with the restrictons $\phi_{0} \in[-\pi / 2, \pi / 2]$ and $\phi_{0} \in[\pi / 2,3 \pi / 2]$, covering the whole parameter space. 


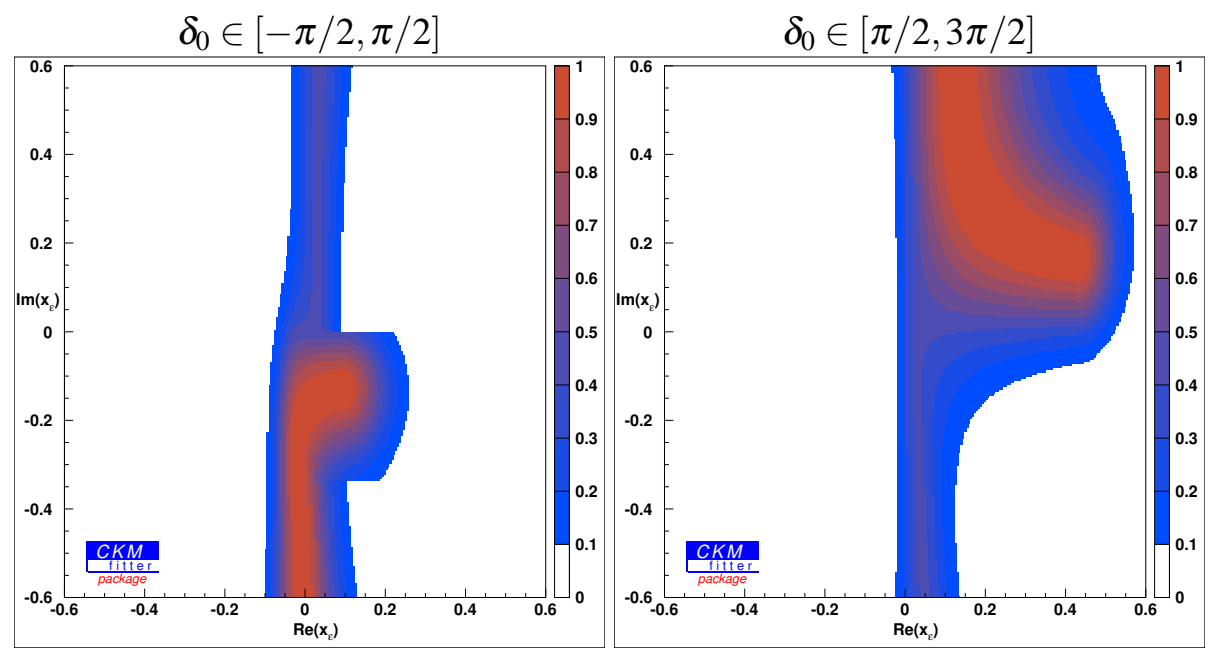

Figure 1: The fit results for the $U$-spin breaking parameters in $B \rightarrow J / \psi K$ and $B \rightarrow J / \psi \pi$.

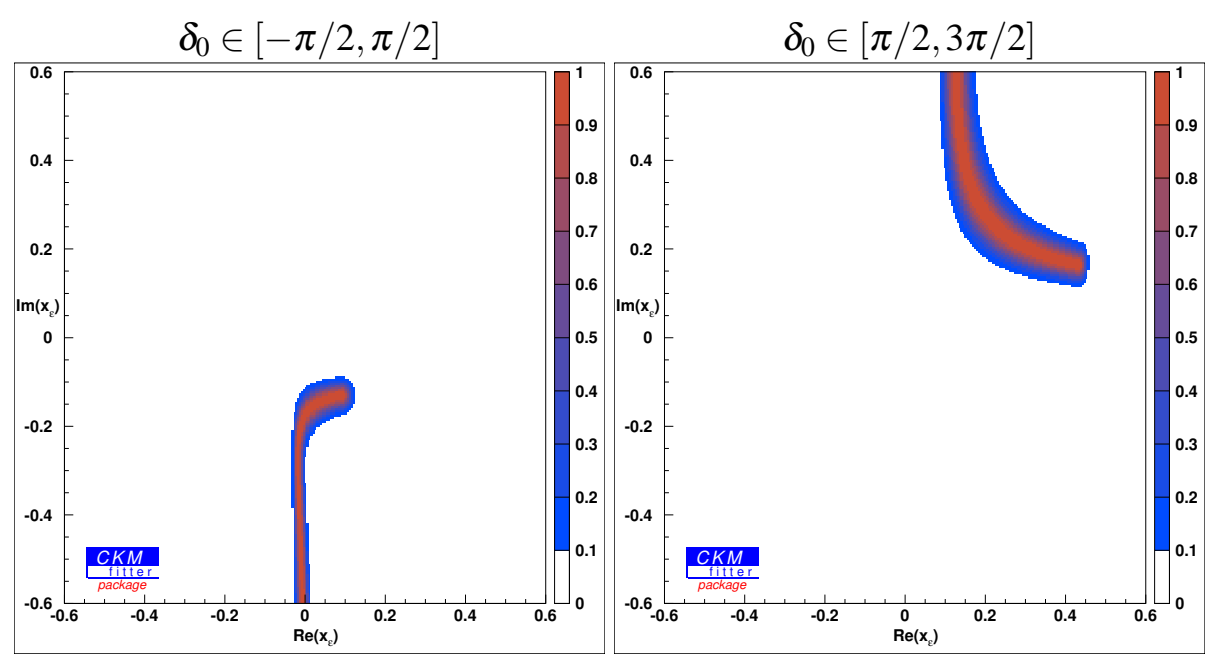

Figure 2: As figure 1, but dividing all experimental uncertainties by five.

In the fit, the non-vanishing central values for the $\mathrm{CP}$ asymmetries imply a non-vanishing value for $r_{0}, r_{0} \geq 0.06 @ 1 \sigma$, in combination with a non-trivial phase. However, as is obvious from the significance of the data, at $2 \sigma$ zero is included in the allowed range. Concerning $U$-spin breaking, the fit prefers a non-vanishing imaginary part of the $U$-spin breaking parameter $x_{\varepsilon}$. This is due to (3.2) showing a deviation from zero, and especially preferring equal signs for the CP asymmetries, while the branching ratios, as seen above, are compatible with no breaking at all. This is again a hint to non-factorizable $U$-spin breaking.

In order to see which of the structures visible in these plots come from the present uncertainties and which are due to the parametrization, the fits are repeated with all experimental uncertainties divided by five. The results are shown in figure 2. In this case, the $\operatorname{sign} \operatorname{of} \operatorname{Im}\left(x_{\varepsilon}\right)$ is correlated uniquely with the value of the strong phase, and, due to the higher significance, $\left|x_{\mathcal{E}}\right|=0$ can be excluded. 


\section{Conclusions}

Since methods based on perturbative QCD do not seem to converge quickly to allow for a reliable prediction for non-leptonic decays, the method of flavour symmetries looks more promising. Clearly the latter will allow us to perform precision calculations only if we get a reasonable control over symmetry breaking.

Using the full $S U(3)$ flavour symmetry becomes quite complicated once its breaking is taken into account. However, isospin may be assumed to be a reasonably good symmetry and hence only the breaking along the "orthogonal" directions in $S U(3)$ space has to be considered. Here, the $U$-spin subgroup of $S U(3)$ has been studied, under which the weak Hamiltonian for $b$ decays as well as the breaking term have a simple structure. Based on this it has been discussed how $U$-spin breaking can be incorporated on a purely group theoretical basis, with a first application given by $B^{-} \rightarrow J / \psi K(\pi)$. Here, not surprisingly, large deviations from the naive factorization limit have been observed, with a different size and a non-vanishing imaginary part preferred.

However, the full strength of this approach can be exploited only in the future. Since the $B_{d}$ and the $B_{s}$ form a $U$-spin doublet, it requires information on decay modes which will be gathered in the near future at the LHC. With a sufficient amount of data there will be a chance to obtain control over flavour $S U$ (3) breaking and hence a possible road to precise predictions for nonleptonic decays may be opened.

\section{Acknowledgements}

This work was supported in part by the EU MRTN-CT-2006-035482 (FLAVIAnet), by MICINN (Spain) under grant FPA2007-60323, and by the Spanish Consolider-Ingenio 2010 Programme CPAN (CSD2007-00042), and by the german research foundation DFG under contracts no. MA1187/10-1 and KH205/1-1.

\section{References}

[1] A. Soni and D.A. Suprun, Determination of gamma from charmless $B->M(1) M(2)$ decays using U-spin, Phys. Rev. D75 (2007) 054006 [hep-ph/ 0609089 ].

[2] M. Jung and Th. Mannel, General Analysis of U-Spin Breaking in B Decays, SI-HEP (2009) 2009-01

[3] R. Fleischer, New strategies to extract beta and gamma from $\mathrm{B} / \mathrm{d}->\mathrm{pi}+$ pi- and $\mathrm{B} / \mathrm{s}->\mathrm{K}+\mathrm{K}-$, Phys. Lett. B459 (1999) 306 [hep-ph/9903456].

M. Gronau, U-spin symmetry in charmless B decays, Phys. Lett. B492 (2000) 297 [hep-ph/0008292].

[4] C. Amsler et al. (Particle Data Group), Review of particle physics, Phys. Lett. B667 (2008) 1.

[5] G. Duplancic, A. Khodjamirian, T. Mannel, B. Melic and N. Offen, Light-cone sum rules for $B \rightarrow \pi$ form factors revisited, JHEP 0804 (2008) 014 [0801 . 1796 [hep-ph] ].

G. Duplancic and B. Melic, B, Bs -> K form factors: an update of light-cone sum rule results, Phys. Rev. D 78, 054015 (2008) [0805.4170 [hep-ph] ].

[6] D. Becirevic and A.B. Kaidalov, Comment on the heavy $\rightarrow>$ light form factors, Phys. Lett. B478 (2000) 417 [hep-ph/9904490]. 
[7] J. Charles et al. (CKMfitter Group), CP violation and the CKM matrix: Assessing the impact of the asymmetric B factories, Eur. Phys. J. C41 (2005) 1 [hep-ph/ 0406184 ], Updated results and plots available at: http: / / ckmfitter.in2p3.fr. 